\author{
基于新型电子受体 1,3,5-三苯酰基苯的两种热活化延迟 \\ 荧光材料的合成及性能研究 \\ 王志强*, ${ }^{*}$ 白美丹 ${ }^{b}$ 张明 $b$ 张智强*,c 冯勋 ${ }^{a}$ 郑才俊*,b \\ ( ${ }^{a}$ 洛阳师范学院化学化工学院 河南省功能导向多孔材料重点实验室 洛阳 471934) \\ ( ${ }^{b}$ 电子科技大学光电科学与工程学院 成都 610054) \\ ( ${ }^{c}$ 郑州轻工业大学材料与化学工程学院 郑州 450001)
}

\begin{abstract}
摘要 以 $1,3,5$-三苯酰基苯(TBP)作为电子受体, 1,8-二甲基味唑( $\mathrm{DmCz}$ )和 1,3,6,8-四甲基味唑( $\mathrm{TmCz}$ )分别作为电子给体, 合成了两种新的热活化延迟苂光材料 TBP-DmCz 和 TBP-TmCz. 热重和差热测试结果表明, 这两种材料都具有高热稳 定性. 理论计算显示, 材料的最高电子占据轨道和最低电子未占轨道分别分布在味唑和 1,3,5-三苯酰基苯结构单元上, 两种分子轨道几乎没有重叠, 具有热活化延迟荧光材料分子轨道的典型特征. 分子轨道能级测算结果显示, 增加味唑 结构单元上甲基的数量, 能明显升高材料的最高电子占据轨道能级. TBP-DmCz 和 TBP-TmCz 的最低激发单重态和最 低激发三重态间能级差 $\left(\Delta E_{\mathrm{ST}}\right.$ ) 都非常小, 分别为 0.05 和 $0.01 \mathrm{eV}$. 在甲苯溶液中, 两种材料均表现出了明显的分子内电 荷转移跃迁吸收, TBP-DmCz 和 TBP-TmCz 的发光峰分别出现在 488 和 $502 \mathrm{~nm}$. 用 TBP-DmCz 和 TBP-TmCz 作为掺杂 客体材料制备出了两种高性能电致发光器件, 器件的最大外量子效率分别达到了 $13.6 \%$ 和 $18.3 \%$.

关键词 电致发光; 发光材料; 热活化延迟苂光; $1,3,5$-三苯酰基苯; 咔唑
\end{abstract}

\title{
Synthesis and Properties of Two Novel Thermally Activated Delayed Fluorescence Materials with 1,3,5-Tribenzoylbenzene as Electron-Acceptor
}

\author{
Wang, Zhiqiang*,a \\ Zhang, Zhiqiang*,c \\ Bai, Meidan ${ }^{b}$ \\ Zhang, Ming ${ }^{b}$ \\ Feng, Xun ${ }^{a}$ \\ Zheng, Caijun*,b \\ ( ${ }^{a}$ College of Chemistry and Chemical Engineering and Henan Key Laboratory of Function-Oriented Porous \\ Materials, Luoyang Normal University, Luoyang 471934) \\ $\left({ }^{b}\right.$ School of Optoelectronic Science and Engineering, University of Electronic Science and Technology of \\ China (UESTC), Chengdu 610054) \\ ( ${ }^{c}$ Department of Material and Chemical Engineering, Zhengzhou University of Light Industry, Zhengzhou 450001)
}

\begin{abstract}
Two thermally activated delayed fluorescence (TADF) materials TBP-DmCz and TBP-TmCz were successfully synthesized using 1,3,5-tribenzoylbenzene (TBP) as electron-acceptor, 1,8-dimethylcarbazole (DmCz) and 1,3,6,8-tetramethylcarbazole $(\mathrm{TmCz})$ as electron-donor, respectively. Thermal gravimetric analysis show that the thermal decomposition temperatures $\left(T_{\mathrm{d}}\right)$ are $479{ }^{\circ} \mathrm{C}$ for TBP-DmCz and $484{ }^{\circ} \mathrm{C}$ for TBP-TmCz and no glass transition was found for both materials during the differential scanning calorimetry investigations. The highest occupied molecular orbitals (HOMO) are confined on the carbazole unit, while the lowest unoccupied molecular orbitals (LUMO) are located on the 1,3,5-tribenzoylbenzene unit, and there is almost no overlap between HOMO and LUMO, which is the typical molecular orbital character of TADF materials. Meanwhile, TBP-DmCz and TBP-TmCz possess degenerated HOMO and LUMO, which would promote the radiative transitions as the transitions could take place from all degenerated LUMOs to HOMOs. The HOMO level of TBP-TmCz is obviously higher than that of TBP-DmCz due to increasing the number of methyl groups at the electron-donor carbazole, and the LUMO levels of TBP-DmCz and TBP-TmCz only show a small difference because these materials have the same electron-acceptor 1,3,5-tribenzoylbenzene. In toluene solution, these materials have very similar absorption spectra and exhibit absorption bands assigned to intramolecular charge-transfer transition. The spectral peaks are located at $488 \mathrm{~nm}$ for TBP-DmCz and $502 \mathrm{~nm}$ for TBP-TmCz, respectively, in toluene solution at room temperature. According to the fluorescence and phosphorescence spectra of these materials in 1,3-bis $(N$-carbazolyl)benzene $(\mathrm{mCP})$ film at $77 \mathrm{~K}$, the energy gaps between the lowest singlet and triplet $\left(\Delta E_{\mathrm{ST}}\right)$ of TBP-DmCz and TBP-TmCz are calculated to be 0.05 and $0.01 \mathrm{eV}$, respectively. The fluorescence decay behaviors at different temperatures $(100,200$ and $300 \mathrm{~K})$ proved that emissions of TBP-DmCz and TBP-TmCz contain TADF component. The electroluminescence devices with TBP-DmCz and TBP-TmCz as the emitters
\end{abstract}

*E-mail: wzq197811@1ynu.edu.cn; zhangzq@zzuli.edu.cn; zhengcaijun@uestc.edu.cn

Received October 16, 2019; published December 25, 2019.

Supporting information for this article is available free of charge via the Internet at http://sioc-journal.cn.

Project supported by the National Natural Science Foundation of China (No. 51773029) and the Henan Natural Science Foundation (No. 182300410230). 项目受国家自然科学基金(No. 51773029)和河南省自然科学基金(No. 182300410230)资助. 
show high efficiency and low efficiency roll-off. The maximum external quantum efficiencies of devices based on TBP-DmCz and TBP-TmCz are $13.6 \%$ and $18.3 \%$, respectively.

Keywords electroluminescence; light-emitting material; thermally activated delayed fluorescence; 1,3,5-tribenzoylbenzene; carbazole

\section{1 引言}

作为新一代平板显示技术, 有机电致发光二极管 (OLEDs) 具有驱动电压低、发光亮度高、响应速度快、 轻薄和可弯折等优点, 在学术界和产业界受到了广泛关 注 $^{[1 \sim 10]}$. 在 OLEDs 发光过程中, 电激发产生的单重态和 三重态激子数量比为 $1: 3$. 基于苂光材料的 OLEDs 只 能利用单重态激子发光, 器件工作效率普遍较低; 而基 于磷光材料的 OLEDs 则能将单重态和三重态激子全部 转化为可见光, 效率远高于苂光 OLEDs ${ }^{[11 \sim 13]}$. 然而, 目 前具有应用价值的磷光材料均为贵金属配合物, 如 $\operatorname{Ir}(\mathrm{III})$ 和 $\operatorname{Pt}(\mathrm{II})$ 配合物等, 这些金属的价格昂贵, 且资源 有限, 不利于大规模应用这些材料.

自 2012 年 Adachi 课题组 ${ }^{[14]}$ 将热活化延迟苂光 (TADF)材料应用于 OLEDs 以来, 该类材料一直被视为 最具应用前景的电致发光材料 ${ }^{[15 ~ 25]}$. 首先, TADF 材料 的最低激发单重态 $\left(\mathrm{S}_{1}\right)$ 和最低激发三重态 $\left(\mathrm{T}_{1}\right)$ 间能级差 $\left(\Delta E_{\mathrm{ST}}\right)$ 小, 室温下即可发生 $\mathrm{T}_{1} \rightarrow \mathrm{S}_{1}$ 反系间窝跃(RISC), 继而可通过 $\mathrm{S}_{1} \rightarrow \mathrm{S}_{0}$ (基态)辐射跃迁产生 $\mathrm{TADF}^{[26-29]}$. 因 此, 用 TADF 材料制备的 OLEDs 也能利用全部单重态 和三重态激子发光. 其次, 通过合理设计分子结构, 纯 有机化合物以及廉价金属的配合物(如 $\mathrm{Cu}(\mathrm{I})$ 配合物)均 能发射高效率 TADF，相比于贵金属磷光化合物，材料 的制备成本大幅降低 ${ }^{[30 ~ 37]}$. 正是由于上述优点, TADF 材料近年来得到了快速发展, 尤其是纯有机 TADF 材 料 ${ }^{[37 \sim 41]}$.

纯有机 TADF 材料通常由电子给体和电子受体两种 结构单元组成, 材料的最高电子占据轨道 (HOMO) 和最 低电子未占轨道( LUMO) 分别分布在电子给体和受体 上; 而且, 两种分子轨道重叠越少, 材料的 $\Delta E_{\mathrm{ST}}$ 越 小 ${ }^{[37]}$. 目前, 已经有多种类型的电子受体成功应用在 TADF 材料中，包括氧基苯、三芳基嗍烷、二苯基亚砜、 二芳基酮和氮杂环芳香化合物等 ${ }^{[37]}$. 1,3,5-三苯酰基苯 (TBP) 是最近开发的一种新型电子受体, 可用作构筑热 稳定性高的星形 TADF 材料 ${ }^{[42,43]}$; 之前的研究显示, 以 TBP 作为电子受体的 TADF 材料具有多个辐射跃迁通 道, 能提高材料发光效率 ${ }^{[42]}$. 本文以 $\mathrm{TBP}$ 作为电子受 体，1,8-二甲基咔唑和 1,3,6,8-四甲基咔唑分别作为电子 给体, 合成了两种 TADF 材料 TBP-DmCz 和 TBP-TmCz. 这两种材料不仅表现出了高热稳定性, 而且均具有非常 小的 $\Delta E_{\mathrm{ST}}$, 发射光表现出了明显的 TADF 特征. 分别以 TBP-DmCz 和 TBP-TmCz 作为发光层掺杂客体材料, 制 备出了两种高性能电致发光器件, 器件的最大外量子效 率分别达到了 $13.6 \%$ 和 $18.3 \%$.

\section{2 结果与讨论}

\section{1 合成与结构}

材料 TBP-DmCz 和 TBP-TmCz 的合成路线如图式 1 所示. 1,3,5-苯三甲酰氯(1)与溴苯通过 Friedel-Crafts 芳 基化反应得到中间产物 2; 化合物 2 分别与 1,8-二甲基 咔唑(3)、1,3,6,8-四甲基咔唑(4)通过 Buchwald-Hartwig 偶联反应即可获得 TBP-DmCz 和 TBP-TmCz. 化合物结 构通过核磁、质谱以及元素分析进行了表征. 通过密度 泛函理论(DFT)计算研究了 TBP-DmCz 和 TBP-TmCz 的 立体结构和分子轨道. 由于咔唑基团 $1 、 8$ 位上甲基的 位阻作用, 使得这两种材料中的咔唑结构单元和与之相 连的苯环间二面角均接近 $90^{\circ}$ (图 1, 图 S1). 这种高度扭 曲结构可降低电子给体咔唑和电子受体 TBP 间的共轭 程度, 有利于实现 HOMO 与 LUMO 的分离. 计算结果 显示, 两种材料的 $\mathrm{HOMO}$ 均分布在咔唑结构单元上, 而 LUMO 分布在 TBP 结构单元上, 两种分子轨道间几乎 没有重叠, 具有 TADF 材料分子轨道的典型特征. 另外, 这两种材料的 LUMO 和 LUMO+1 分子轨道的能级相同, HOMO、HOMO-1 和 HOMO-2 分子轨道也具有相同的 能级, 分别为两组简并分子轨道(图 1, 图 $\mathrm{S} 1$ ), 计算结果 与文献报道非常相似, 意味着 TBP-DmCz 和 TBP-TmCz 可能具有多个辐射跃迁通道 ${ }^{[42]}$. 计算得到, TBP-DmCz 的 $\mathrm{S}_{1}$ 态能级为 $2.466 \mathrm{eV}, \mathrm{T}_{1}$ 态能级为 $2.462 \mathrm{eV}, \Delta E_{\mathrm{ST}}$ 为 $0.004 \mathrm{eV} ; \mathrm{TBP}-\mathrm{TmCz}$ 的 $\mathrm{S}_{1}$ 态能级为 $2.340 \mathrm{eV}$, 理论 $\mathrm{T}_{1}$ 态能级为 $2.334 \mathrm{eV}, \Delta E_{\mathrm{ST}}$ 为 $0.006 \mathrm{eV}$. 极小的 $\Delta E_{\mathrm{ST}}$ 表明 TBP-DmCz 和 TBP-TmCz 预期具有良好的 TADF 特性.

\section{2 热稳定性与电子轨道能级}

热重(TGA)和差示扫描量热(DSC)测试结果显示, 这两种材料都具有高热稳定性。在氮气氛下, TBP-DmCz 和 TBP-TmCz 的热分解温度 $\left(T_{\mathrm{d}}\right.$, 失重 $5 \%$ ) 分别为 479 和 $484{ }^{\circ} \mathrm{C}$ (图 2), 在 $50 \sim 300{ }^{\circ} \mathrm{C}$ 温度范围内, 两种材料均没有发生玻璃化转变现象. 根据循环伏安法 $(C V)$ 测得的氧化电位, 计算得到 TBP-DmCz 和 TBP$\mathrm{TmCz}$ 的 HOMO 能级分别为 -5.70 和 $-5.62 \mathrm{eV}$. 可见, 增加咔唑结构单元上甲基数量, 材料的 HOMO 能级会 明显升高. 这是因为两种材料的 HOMO 轨道均主要分 布在咔唑结构单元上，而具有供电子能力的甲基能升高 分子轨道能级. 根据材料吸收光谱边沿波长, 计算出 TBP-DmCz 和 TBP-TmCz 的 HOMO 与 LUMO 间能级差 分别为 2.75 和 $2.65 \mathrm{eV}$; 结合 $\mathrm{HOMO}$ 能级值, 得到 TBP-DmCz 和 TBP-TmCz 的 LUMO 能级分别为 -2.95 和 $-2.97 \mathrm{eV}$. 由于这两种材料的 LUMO 轨道均分布在 

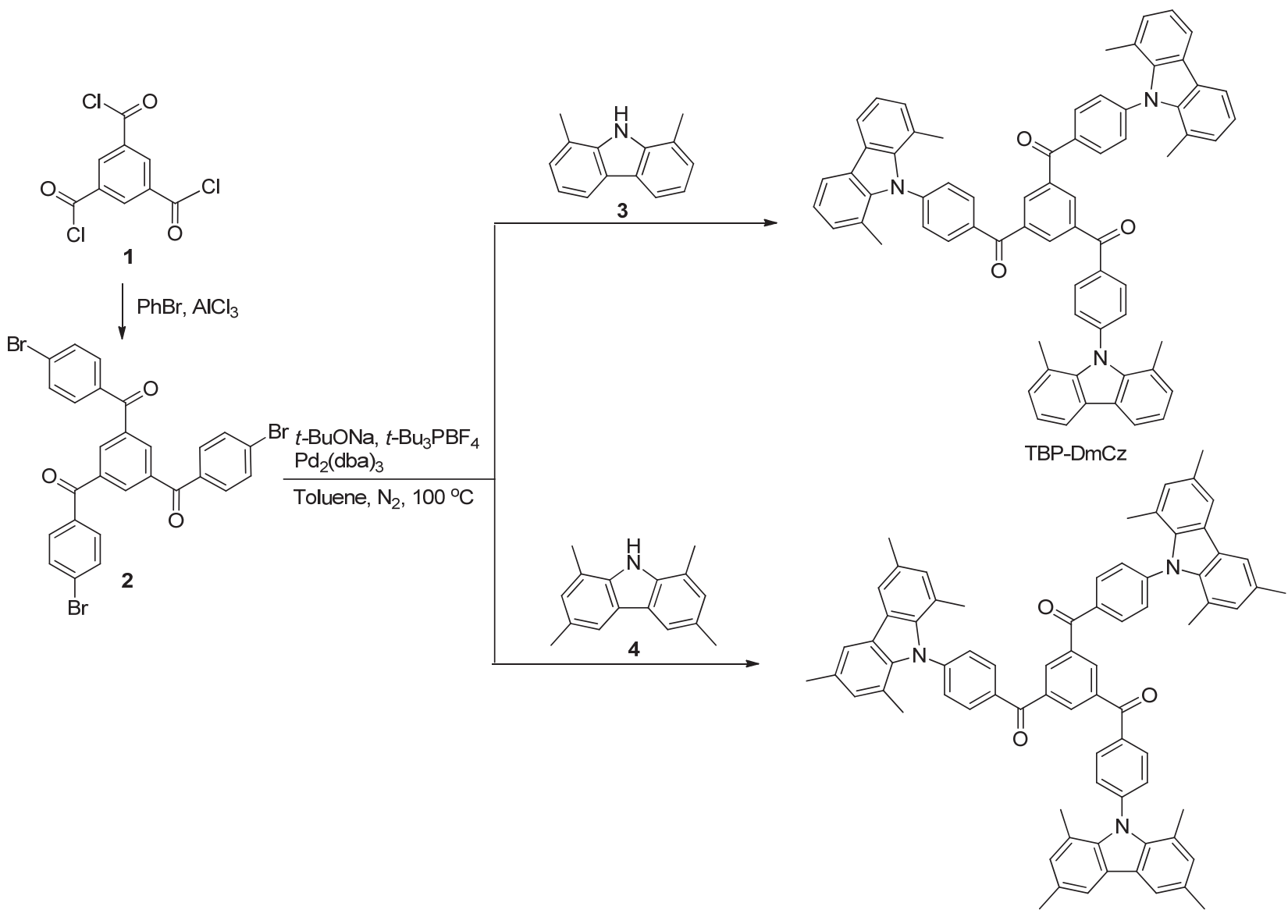

TBP-TmCz

图式 1 TBP-DmCz 和 TBP- $\mathrm{TmCz}$ 的合成路线

Scheme 1 Synthetic routes of TBP-DmCz and TBP-TmCz<smiles>C1=C[As]2C=C[As]12</smiles>

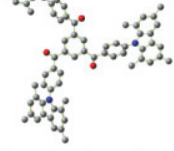

Stereostructure

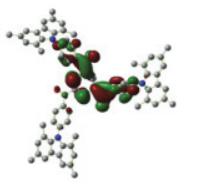

LUMO+1 $(-2.47 \mathrm{eV})$

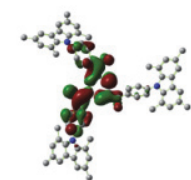

LUMO (-2.47 eV)
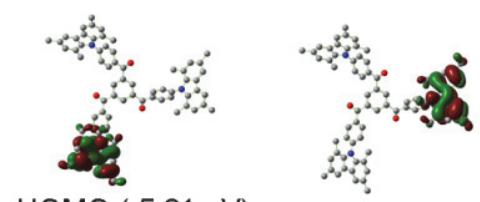

HOMO $(-5.21 \mathrm{eV})$

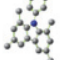

HOMO-1 (-5.21 eV) HOMO-2 (-5.21 eV)

图 1 TBP-TmCz 的立体结构和分子轨道

Figure 1 Stereostructure and molecular orbitals of TBP-TmCz

TBP 结构单元上, 因此它们的 LUMO 能级值差别不大. 表 1 列出了这两种材料的主要物理性能数据.

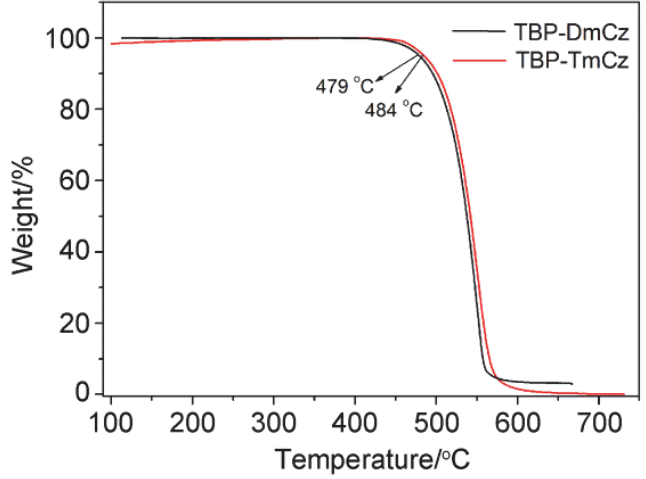

图 $2 \mathrm{TBP}-\mathrm{DmCz}$ 和 $\mathrm{TBP}-\mathrm{TmCz}$ 的 TGA 曲线 Figure 2 TGA curves of TBP-DmCz and TBP-TmCz

\section{3 光物理性能}

图 3 为 TBP-DmCz 和 TBP- $\mathrm{TmCz}$ 在甲苯溶液中的 紫外-可见吸收光谱与光致发光(PL)光谱. 这两种材料 的吸收光谱非常相似; 波长小于 $350 \mathrm{~nm}$ 的强吸收带为 $\pi-\pi$ *跃迁吸收; TBP-DmCz 在 $384 \mathrm{~nm}$ 和 TBP- $\mathrm{TmCz}$ 在 $390 \mathrm{~nm}$ 的弱吸收带可归属为从电子给体咔唑到电子受 体 TBP 间的分子内电荷转移(ICT) 跃迁吸收. 在甲苯溶 
表 $1 \mathrm{TBP}-\mathrm{DmCz}$ 和 $\mathrm{TBP}-\mathrm{TmCz}$ 的物理性能数据

Table 1 Physical properties of TBP-DmCz and TBP-TmCz

\begin{tabular}{ccccccc}
\hline Compound & $\lambda_{\mathrm{Abs}} / \mathrm{nm}$ & $\lambda_{\mathrm{PL}} / \mathrm{nm}$ & $\Phi_{\mathrm{PL}}{ }^{a} / \%$ & $\Delta E_{\mathrm{ST}} / \mathrm{eV}$ & $\mathrm{HOMO} / \mathrm{eV}$ & $T_{\mathrm{d}} /{ }^{\circ} \mathrm{C}$ \\
\hline TBP-DmCz & $334,347,384$ & 488 & 62.3 & 0.05 & -5.70 & -2.95 \\
TBP-TmCz & $332,347,390$ & 502 & 69.6 & 0.01 & -5.62 & -2.97 \\
\hline
\end{tabular}

${ }^{a}$ Measured as a thin film doped in mCP.

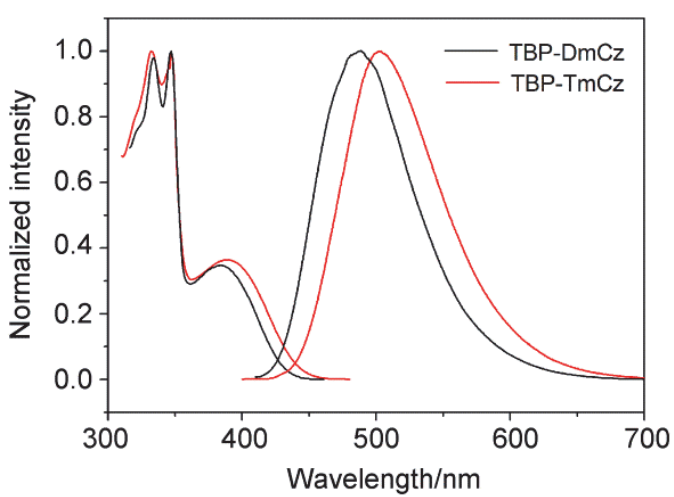

图 $3 \mathrm{TBP}-\mathrm{DmCz}$ 和 $\mathrm{TBP}-\mathrm{TmCz}$ 在甲苯溶液中的吸收光谱与光致发光 (PL)光谱

Figure 3 The absorption and photoluminescence (PL) spectra of TBP-DmCz and TBP-TmCz in toluene solution

剂中, TBP-DmCz 和 TBP-TmCz 的室温发光峰分别出现 在 488 和 $502 \mathrm{~nm}$. 与 TBP-DmCz 相比, TBP-TmCz 的 ICT 吸收带和 PL 光谱均发生了明显红移, 这和材料的分子 轨道能级测试结果一致.

将这两种材料分别与 1,3-二(9-咔唑)苯(mCP)共同 蒸镀, 制备成质量浓度 $(w)$ 为 $5 \%$ 的固态薄膜. TBP$\mathrm{DmCz}$ 和 $\mathrm{TBP}-\mathrm{TmCz}$ 在薄膜中均表现出了较高的光致发 光量子效率 $\left(\Phi_{\mathrm{PL}}\right)$, 分别为 $62.3 \%$ 和 $69.6 \%$. 根据材料在 薄膜中、 $77 \mathrm{~K}$ 温度下的荧光和磷光光谱, 计算出 TBP-DmCz 和 TBP-TmCz 的 $\Delta E_{\mathrm{ST}}$ 分别为 $0.05 \mathrm{eV}$ 和 0.01 $\mathrm{eV}$, 如此小的 $\Delta E_{\mathrm{ST}}$ 意味着材料能够发射高效率的 TADF. TBP-DmCz 和 TBP-TmCz 薄膜在不同温度下的瞬 态苂光衰减测试结果显示(图 4), 两种材料的发射光中 均包含有纳秒级和微秒级寿命的两种组分, 可分别归属 为瞬时苂光和来源于激发三重态的 TADF; 而且, 升高 温度能明显缩短长寿命组分的衰减时间, 这进一步证明 了这两种材料的发射光中存在 TADF.

\section{4 电致发光性能}

用 TBP-DmCz 和 TBP-TmCz 分别作为发光层客体 材料, 通过优化器件结构, 制备出了两种高性能的电致 发光(EL)器件. 分别为器件 I: ITO/TAPC (35 nm)/TCTA $(10 \mathrm{~nm}) / \mathrm{mCP}: \mathrm{TBP}-\mathrm{DmCz}(w=10 \%)(20 \mathrm{~nm}) / \mathrm{TmPyPb}(40$ $\mathrm{nm}) / \mathrm{LiF}(1 \mathrm{~nm}) / \mathrm{Al}$; 器件 II: ITO/TAPC (35 nm)/TCTA $(10 \mathrm{~nm}) / \mathrm{mCP}: \mathrm{TBP}-\mathrm{TmCz}(w=5 \%)(20 \mathrm{~nm}) / \mathrm{TmPyPb}(40$ $\mathrm{nm}) / \mathrm{LiF}(1 \mathrm{~nm}) / \mathrm{Al}$. ITO(氧化铟锡)和 $\mathrm{LiF} / \mathrm{Al}$ 分别为阳极 和阴极; TAPC、 TCTA、 TmPyPb 和 $\mathrm{mCP}$ 的结构如图 5 所示, 分别用作空穴传输层、电子阻挡层、电子传输层
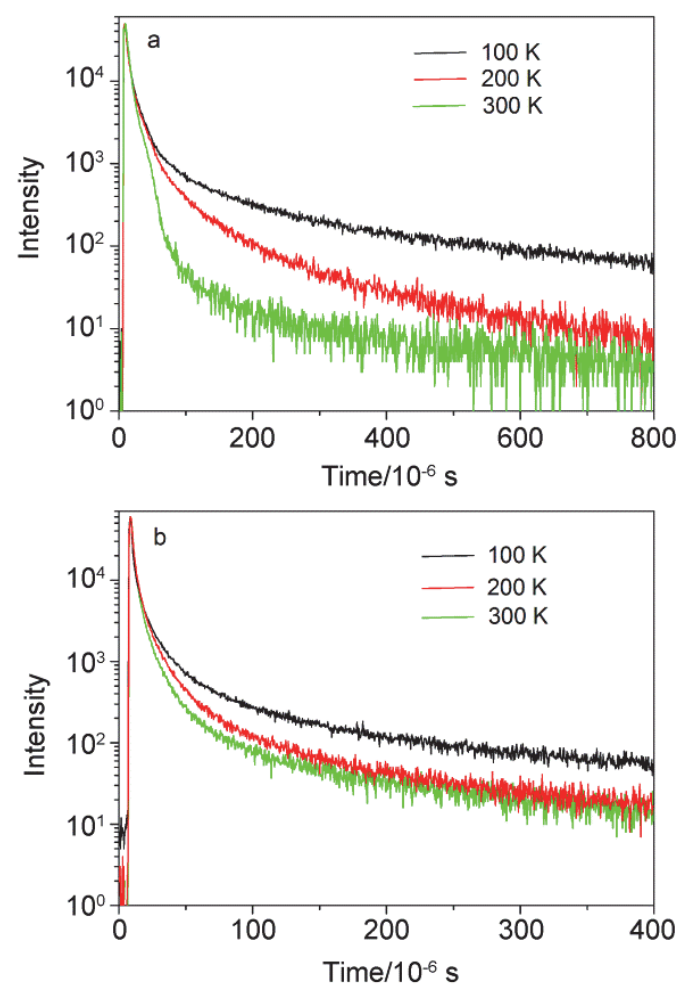

图 4 TBP- $\mathrm{DmCz}(\mathrm{a})$ 和 TBP- $\mathrm{TmCz}$ (b) 在 $\mathrm{mCP}$ 薄膜中不同温度下的荧 光衰减曲线

Figure 4 The temperature-depended transient PL decay curves of TBP-DmCz (a) and TBP-TmCz (b) in mCP film

和发光层主体材料. 表 2 列出了电致发光器件 I 和 II 的 主要性能数据.

如图 6 所示, 器件 I 和 II 的发光峰分别位于 496 和 $508 \mathrm{~nm}$, 相比于 TBP-DmCz 和 TBP-TmCz 的 PL 光谱仅 发生了少量红移. 器件 I 和 II 均表现出了低开启电压和 高发光亮度, 开启电压均为 $3.1 \mathrm{~V}$, 最大发光亮度分别达 到了 $10810 \mathrm{~cd} / \mathrm{m}^{2}$ 和 $10940 \mathrm{~cd} / \mathrm{m}^{2}$ (图 7). 两种器件的发光 外量子效率都超过了传统苂光 OLEDs 的理论极限值 (5\%), 器件 I 和 II 的最大外量子效率分别为 $13.6 \%$ 和 $18.3 \%$, 最大功率效率分别为 $33.8 \mathrm{~lm} / \mathrm{W}$ 和 $47.7 \mathrm{~lm} / \mathrm{W}$ (图 8). 而且, 随着发光亮度升高, 发光效率只发生了小 幅下降, 发光亮度为 $1000 \mathrm{~cd} / \mathrm{m}^{2}$ 时, 器件 I 和 II 仍分别 具备 $10.9 \%$ 和 $14.2 \%$ 的外量子效率, 说明 TBP-DmCz 和 $\mathrm{TBP}-\mathrm{TmCz}$ 具有较高的实际应用价值.

\section{3 结论}

利用 1,3,5-三苯酰基苯和甲基咔唑分别作为电子受 

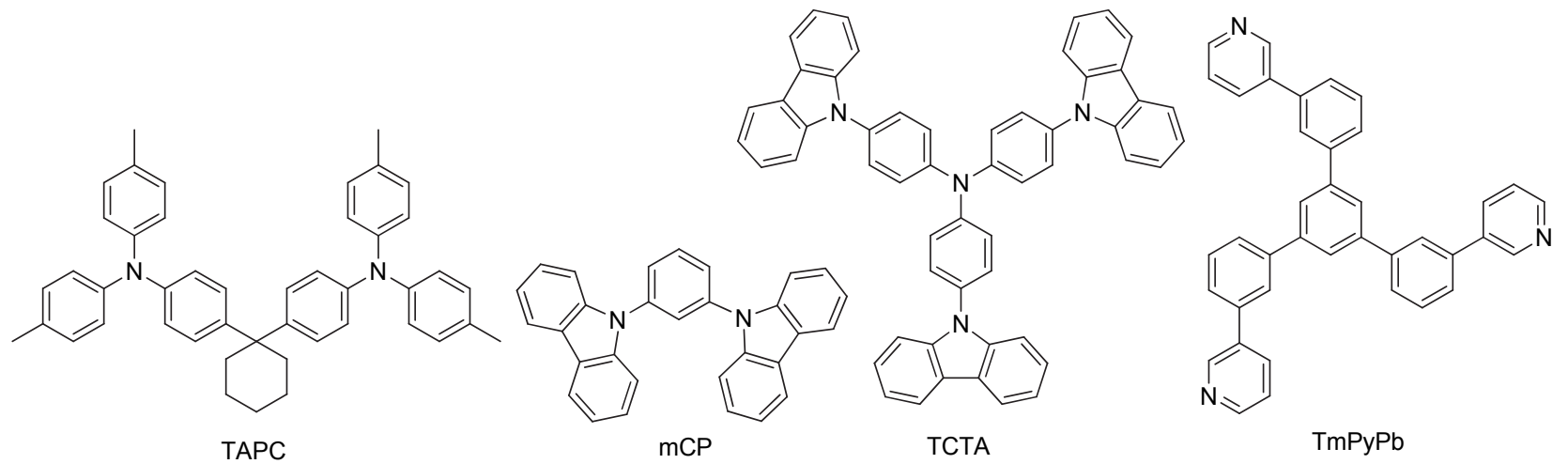

图 $5 \mathrm{TAPC}, \mathrm{TCTA}, \mathrm{TmPyPb}$ 和 $\mathrm{mCP}$ 的分子结构

Figure 5 Molecular structures of TAPC, TCTA, TmPyPb and $\mathrm{mCP}$

表 2 电致发光器件 I 和 II 的性能数据

Table 2 The EL performance data of devices I and II

\begin{tabular}{ccccccc}
\hline Devices (guest) & $V_{\mathrm{on}}{ }^{a} / \mathrm{V}$ & $\lambda_{\mathrm{EL}} / \mathrm{nm}$ & $L_{\max }{ }^{b} /\left(\mathrm{cd} \cdot \mathrm{m}^{-2}\right)$ & $\mathrm{CE}_{\max }{ }^{c} /\left(\mathrm{cd} \cdot \mathrm{A}^{-1}\right)$ & $\mathrm{PE}_{\max }{ }^{d} /\left(\operatorname{lm} \cdot \mathrm{W}^{-1}\right)$ & $\mathrm{EQE}_{\max }{ }^{e} / \%$ \\
\hline I (TBP-DmCz) & 3.1 & 496 & 10810 & 33.4 & 33.8 & 13.6 \\
II (TBP-TmCz) & 3.1 & 508 & 10940 & 50.1 & 47.7 & 18.3 \\
\hline
\end{tabular}

${ }^{a}$ Turn-on voltage measured at the luminance of $1 \mathrm{~cd} / \mathrm{m}^{2} ;{ }^{b}$ maximum luminance; ${ }^{c}$ maximum current efficiency; ${ }^{d}$ maximum power efficiency; ${ }^{e}$ maximum external quantum efficiency.

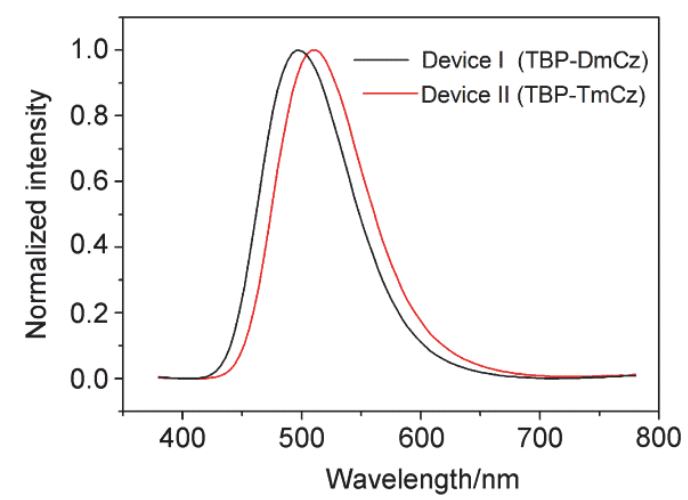

图 6 器件 I 和 II 的电致发光光谱

Figure 6 Electroluminescence spectra of devices I and II

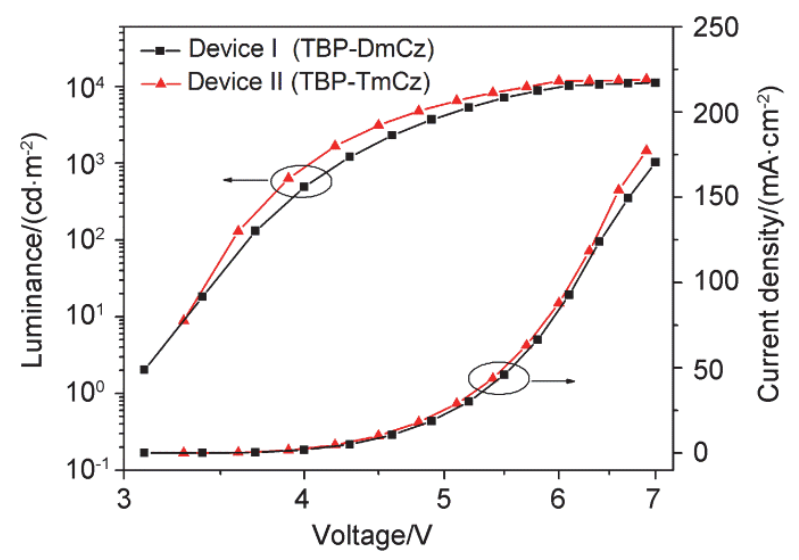

图 7 器件 I 和 II 的电流密度-电压-亮度曲线

Figure 7 The current density-voltage-luminance characteristics of devices I and II

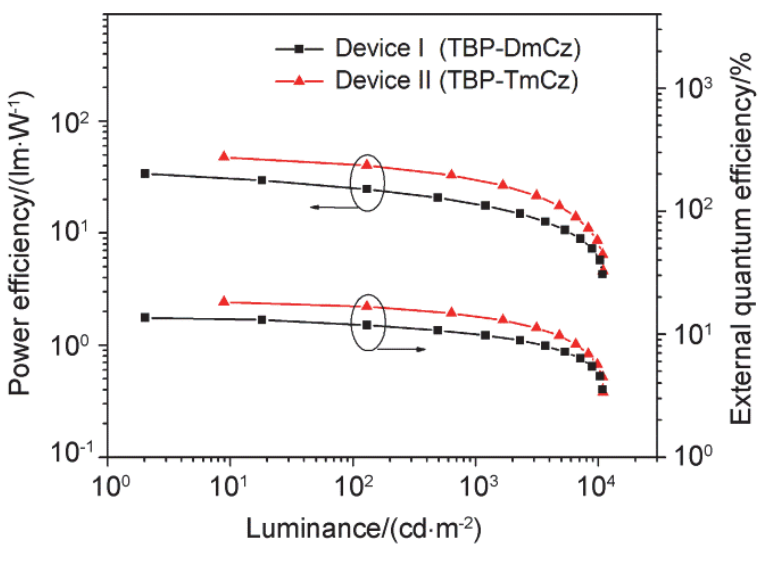

图 8 器件 I 和 II 的功率效率-外量子效率-亮度曲线

Figure 8 The power efficiency-external quantum efficiency-luminance characteristics of devices I and II

体和电子给体，合成了两种高热稳定性的 TADF 材料 TBP-DmCz 和 TBP-TmCz. 材料的 HOMO 和 LUMO 分 子轨道高度分离, 分别分布在电子给体和受体上, 显示 出了 $\mathrm{TADF}$ 材料分子轨道的典型特征. $\mathrm{TBP}-\mathrm{DmCz}$ 和 $\mathrm{TBP}-\mathrm{TmCz}$ 均具有非常小的 $\Delta E_{\mathrm{ST}}$, 瞬态荧光衰减测试结 果证实了这两种材料的发射光中包含 TADF 组分. 分别 用 TBP-DmCz 和 TBP-TmCz 作为发光层客体材料, 制备 出了性能优异的电致发光器件. 两种器件均表现出了低 开启电压、高发光亮度和高发光效率; 并且, 在达到实 际应用水平的亮度 $\left(1000 \mathrm{~cd} / \mathrm{m}^{2}\right)$ 时, 器件仍能保持较高 的发光效率. 另外, 与报道的其它 TADF 材料相比, TBP-DmCz 和 TBP-TmCz 中的 1,3,5-三苯酰基苯和甲基 咔唑基团是更为常用和简单的化学基团, 能为材料的合 
成与应用提供便利. TBP-DmCz 和 TBP-TmCz 的研究也 可为相关基团在 TADF 材料中的应用提供可行的思路.

\section{4 实验部分}

\section{1 材料的合成}

\section{1 .1 化合物 2 的合成}

氮气保护下, 在烧瓶中加入 $2.65 \mathrm{~g}(10 \mathrm{mmol}) 1,3,5-$ 苯三甲酰氯(1)和 $12.56 \mathrm{~g}(80 \mathrm{mmol})$ 溴苯, 冰浴中搅拌 15 $\mathrm{min}$; 然后, 缓慢加入 $1.07 \mathrm{~g}(8 \mathrm{mmol})$ 无水 $\mathrm{AlCl}_{3}$, 室温 搅拌 $12 \mathrm{~h}$, 缓慢升温至 $90{ }^{\circ} \mathrm{C}$, 继续反应 $2 \mathrm{~h}$. 待反应液 冷却至室温, 加入 $200 \mathrm{~mL}$ 蒸馏水, 抽滤, 用蒸馏水洗涤 固体. 粗产品用柱层析法提纯, 以体积比为 $1: 1$ 的二氯 甲烷和石油醚混合溶液作为淋洗液, 得到 $5.67 \mathrm{~g}$ 白色固 体(产率 90\%). ${ }^{1} \mathrm{H}$ NMR $\left(400 \mathrm{MHz}, \mathrm{DMSO}-d_{6}\right) \delta: 8.25$ (s, $3 \mathrm{H}), \quad 7.85 \sim 7.77(\mathrm{~m}, 12 \mathrm{H})$. EI-MS $\mathrm{m} / z$ : calcd for $\mathrm{C}_{27} \mathrm{H}_{15} \mathrm{Br}_{3} \mathrm{O}_{3} 627.85$, found 627.30 .

\subsubsection{TBP-DmCz 的合成}

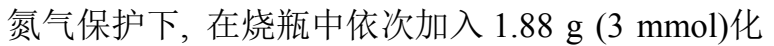
合物 2、1.95 g (10 mmol), 1,8-二甲基咔唑(3)、435 mg (1.5 $\mathrm{mmol})$ 三叔丁基膦四氟喼酸盐、 $1.44 \mathrm{~g}(15 \mathrm{mmol})$ 叔丁醇 钠和 $275 \mathrm{mg}(0.3 \mathrm{mmol})$ 三(二亚苄基丙酮)二钯; 然后, 加入 $100 \mathrm{~mL}$ 无水无氧甲苯作为溶剂, $100{ }^{\circ} \mathrm{C}$ 回流反应 $24 \mathrm{~h}$. 待反应液冷却至室温, 蒸出溶剂, 粗产品用柱层 析法提纯, 以体积比为 $2: 1$ 的二氯甲烷和石油醚混合 溶液作为淋洗液, 得到 $1.96 \mathrm{~g}$ 淡黄色固体产率 $67 \%$. ${ }^{1} \mathrm{H}$ NMR (400 MHz, $\left.\mathrm{CDCl}_{3}\right) \delta: 8.57(\mathrm{~s}, 3 \mathrm{H}), 8.03 \sim 7.99(\mathrm{~m}$, $12 \mathrm{H}), 7.70$ (d, $J=8.4 \mathrm{~Hz}, 6 \mathrm{H}), 7.18$ (t, $J=7.5 \mathrm{~Hz}, 6 \mathrm{H})$, 7.07 (dt, $J=7.2,1.1 \mathrm{~Hz}, 6 \mathrm{H}), 1.93(\mathrm{~s}, 18 \mathrm{H}) ;{ }^{13} \mathrm{C} \mathrm{NMR}(100$ $\left.\mathrm{MHz}, \mathrm{CDCl}_{3}\right) \delta: 193.58,140.55,138.17,136.23,134.19$, $131.85,130.06,129.17,124.33,121.23,120.39,118.16$, 19.81. EI-MS $m / z$ : calcd for $\mathrm{C}_{69} \mathrm{H}_{51} \mathrm{~N}_{3} \mathrm{O}_{3}$ 969.39; found 970.19. Anal. calcd for $\mathrm{C}_{69} \mathrm{H}_{51} \mathrm{~N}_{3} \mathrm{O}_{3}: \mathrm{C} 85.42, \mathrm{H} 5.30, \mathrm{~N}$ 4.33; found C 85.21, H 5.25, N 4.36.

\subsubsection{TBP- $\mathrm{TmCz}$ 的合成}

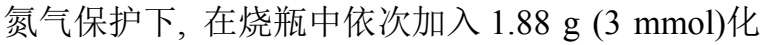
合物 2、2.23 g (10 mmol) 1,3,6,8-四甲基咔唑(4)、435 mg (1.5 mmol)三叔丁基膦四氟嗍酸盐、 $1.44 \mathrm{~g}(15 \mathrm{mmol})$ 叔 丁醇钠和 $275 \mathrm{mg}(0.3 \mathrm{mmol})$ 三(二亚苄基丙酮)二钯; 然 后, 加入 $100 \mathrm{~mL}$ 无水无氧甲苯作为溶剂, $100{ }^{\circ} \mathrm{C}$ 回流反 应 $24 \mathrm{~h}$. 待反应液冷却至室温, 蒸出溶剂, 粗产品用柱 层析法提纯, 以体积比为 $2: 1$ 的二氯甲烷和石油醚混 合溶液作为淋洗液, 得到 $2.47 \mathrm{~g}$ 淡黄色固体(产率 78\%). ${ }^{1} \mathrm{H}$ NMR (400 MHz, $\mathrm{CDCl}_{3}$ ) $\delta: 8.54$ (s, 3H), 7.98 (d, $J=$ $8.0 \mathrm{~Hz}, 6 \mathrm{H}), 7.74$ (s, 6H), $7.66(\mathrm{~d}, J=12.0 \mathrm{~Hz}, 6 \mathrm{H}), 6.87$ $(\mathrm{s}, 6 \mathrm{H}), 2.46(\mathrm{~s}, 18 \mathrm{H}), 1.86(\mathrm{~s}, 18 \mathrm{H}) .{ }^{13} \mathrm{C} \mathrm{NMR}(100 \mathrm{MHz}$, $\left.\mathrm{CDCl}_{3}\right) \delta: 193.63,147.61,139.46,138.19,136.00,134.13$, $131.68,130.40,130.05,129.59,124.56,120.98,117.94$,
21.07, 19.66. EI-MS $m / z$ : calcd for $\mathrm{C}_{75} \mathrm{H}_{63} \mathrm{~N}_{3} \mathrm{O}_{3}$ 1053.49, found 1054.35. Anal. calcd for $\mathrm{C}_{75} \mathrm{H}_{63} \mathrm{~N}_{3} \mathrm{O}_{3}$ : C 85.44, $\mathrm{H}$ 6.02, N 3.99; found C 85.16, H 6.07, N 3.96.

\section{2 电致发光器件的制备与性能测试}

将 ITO 玻璃基片(15 $\Omega$ )依次用无水乙醇、丙酮和去 离子水超声清洗 $5 \mathrm{~min}$, 然后 $120{ }^{\circ} \mathrm{C}$ 烘干, 最后用紫外 臭氧清洗仪处理 $5 \mathrm{~min}$. 真空条件下 $\left(5 \times 10^{-4} \mathrm{~Pa}\right)$, 首先 在清洗过的基片上蒸镀各有机层, 蒸镀速率为 $1 \sim 2 \AA / \mathrm{s}$; 然后, 蒸镀 $\mathrm{LiF}$ 和 $\mathrm{Al}$ 电极层, 蒸镀速率分别为 1 和 10 $\AA / s$. 电致发光光谱和 CIE 色坐标用 PR650 光谱仪测定, 电流、电压和发光亮度分别用 Keithley 2400、R6145 电 压计和 LS-110 亮度计测定. 器件性能测试均由计算机 控制，在室温、常压和空气环境中进行.

\section{References}

[1] Sun, Y. R.; Giebink, N. C.; Kanno, H.; Ma, B. W.; Thompson, M. E.; Forrest, S. R. Nature 2006, 440, 908.

[2] Reineke, S.; Lindner, F.; Schwartz, G.; Seidler, N.; Walzer, K.; Lussem, B.; Leo, K. Nature 2009, 459, 234.

[3] Helander, M. G.; Wang, Z. B.; Qiu, J.; Greiner, M. T.; Puzzo, D. P.; Liu, Z. W. Science 2011, 332, 944.

[4] Han, T.-H.; Lee, Y.; Choi, M.-R.; Woo, S.-H.; Hong, B. H.; Ahn, J.-H.; Lee, T.-W. Nat. Photonics 2012, 459, 105.

[5] Sasabe, H.; Kido, J. J. Mater. Chem. C 2013, 1, 1699.

[6] Chen, S.; Dai, J.; Zhou, K.; Luo, Y.; Su, S.; Pu, X.; Huang, Y.; Lu, Z. Acta Chim. Sinica 2017, 75, 367. (陈仕琦, 代军, 周凯峰, 罗艳菊, 苏仕健, 蒲雪梅, 黄艳, 卢志云, 化学学报, 2017, 75, 367.)

[7] Qiu, Z.; Tan, J.; Cai, N.; Wang, K.; Ji, S.; Huo, Y. Chin. J. Org. Chem. 2019, 39, 679. (邱志鹏, 谭继华, 蔡宁, 王凯, 籍少敏, 霍 延平, 有机化学, 2019, 39, 679.)

[8] He, X.; Xiao, Y.; Yuan, X.; Ye, S.; Jiang, H. Chin. J. Org. Chem. 2019, 39, 761. (何煦, 肖燏萍, 袁釒否, 叶尚辉, 姜鸿基, 有机化 学, 2019, 39, 761.)

[9] Wang, F.; Cao, X.; Mei, L.; Zhang, X.; Hu, J.; Tao, Y. Chin. J. Chem. 2018, 36, 241.

[10] Zhou, W.; Liu, Z.; Wang, Z.; Hu, S.; Liang, A. Chin. J. Org. Chem. 2019, 39, 1214. (周文静，刘志谦，王志平，胡斯帆，梁爱辉，有机 化学, 2019, 39, 1214.)

[11] Xu, H.; Chen, R.; Sun, Q.; Huang, W.; Liu, X. Chem. Soc. Rev. 2014, 43, 3259.

[12] Volz, D.; Wallesch, M.; Fléchon, C.; Danz, M.; Verma, A.; Navarro, J. M.; Zink, D. M.; Bräse, S.; Baumann, T. Green Chem. 2015, 17, 1988

[13] Chi, Y.; Tong, B.; Chou, P.-T. Coord. Chem. Rev. 2014, 281, 1.

[14] Uoyama, H.; Goushi, K.; Shizu, K.; Nomura, H.; Adachi, C. Nature 2012, 492, 234.

[15] Dias, F. B.; Bourdakos, K. N.; Jankus, V.; Moss, K. C.; Kamtekar, K. T.; Bhalla, V.; Santos, J.; Bryce, M. R.; Monkman, A. P. Adv. Mater. 2013, 25, 3707.

[16] Zhang, D. D.; Duan, L. A.; Li, Y. L.; Zhang, D. Q.; Qiu, Y. J. Mater. Chem. C 2014, 2, 8191.

[17] Wang, H.; Xie, L.; Peng, Q.; Meng, L.; Wang, Y.; Yi, Y.; Wang, P. Adv. Mater. 2014, 26, 5198.

[18] Mei, L.; Hu, J.; Cao, X.; Wang, F.; Zheng, C.; Tao, Y.; Zhang, X.; Huang, W. Chem. Commun. 2015, 51, 13024.

[19] Cai, X.; Li, X.; Xie, G.; He, Z.; Gao, K.; Liu, K.; Chen, D.; Cao, Y.; Su, S. J. Chem. Sci. 2016, 7, 4264.

[20] Meng, L.; Wang, H.; Wei, X.; Liu, J.; Chen, Y.; Kong, X.; Lv, X.; Wang, P.; Wang, Y. ACS Appl. Mater. Interfaces 2016, 8, 20955.

[21] Wang, K.; Zheng, C. J.; Liu, W.; Liang, K.; Shi, Y. Z.; Tao, S. L.; Lee, C. S.; Ou, X. M.; Zhang, X. H. Adv. Mater. 2017, 29, 1701476.

[22] Lee, J.; Aizawa, N.; Yasuda, T. Chem. Mater. 2017, 29, 8012.

[23] Shi, Y. Z.; Wang, K.; Li, X.; Dai, G. L.; Liu, W.; Ke, K.; Zhang, M.; Tao, S. L.; Zheng, C. J.; Ou, X. M.; Zhang, X. H. Angew. Chem., Int. Ed. 2018, 57, 9480 .

[24] Yu, L.; Wu, Z.; Xie, G.; Zeng, W.; Ma, D.; Yang, C. Chem. Sci. 2018, 9, 
1385.

[25] Yang, Z.; Mao, Z.; Xu, C.; Chen, X.; Zhao, J.; Yang, Z.; Zhang, Y.; Wu, W.; Jiao, S.; Liu, Y.; Aldred, M. P.; Chi, Z. Chem. Sci. 2019, 10, 8129.

[26] Zhang, M.; Liu, W.; Zheng, C. J.; Wang, K.; Shi, Y. Z.; Li, X.; Lin, H.; Tao, S. L.; Zhang, X. H. Adv. Sci. 2019, 6, 1801938.

[27] Rajamalli, P.; Senthilkumar, N.; Gandeepan, P.; Huang, P. Y.; Huang, M. J.; Ren-Wu, C. Z.; Yang, C. Y.; Chiu, M. J.; Chu, L. K.; Lin, H. W.; Cheng, C. H. J. Am. Chem. Soc. 2016, 138, 628.

[28] Xie, Z.; Chen, C.; Xu, S.; Li, J.; Zhang, Y.; Liu, S.; Xu, J.; Chi, Z. Angew. Chem., Int. Ed. 2015, 54, 7181.

[29] Nikolaenko, A. E.; Cass, M.; Bourcet, F.; Mohamad, D.; Roberts, M. Adv. Mater. 2015, 27, 7236 .

[30] Di, D.; Romanov, A. S.; Yang, L.; Richter, J. M.; Rivett, J. P. H.; Jones, S.; Thomas, T. H.; Jalebi, M. A.; Friend, R. H.; Linnolahti, M.; Bochmann, M.; Credgington, D. Science 2017, 356, 159.

[31] Wang, Z.; Zheng, C.; Wang, W.; Xu, C.; Ji, B.; Zhang, X. Inorg. Chem. 2016, 55, 2157.

[32] Wang, Z.; Sun, X.; Xu, C.; Ji, B. Front. Chem. 2019, DOI: 10. 3389/fchem. 2019.00422

[33] Song, X.; Zhang, D.; Lu, Y.; Yin, C.; Duan, L. Adv. Mater. 2019, 31, 1901923.
[34] Kretzschmar, A.; Patze, C.; Schwaebel, S. T.; Bunz, U. H. F. J. Org. Chem. 2015, 80, 9126.

[35] Zhu, Y.; Zhang, Y.; Yao, B.; Wang, Y.; Zhang, Z.; Zhan, H.; Zhang, B.; Xie, Z.; Wang, Y.; Cheng, Y. Macromolecules 2016, 49, 4373.

[36] Wang, Z.; Cai, J.; Zhang, M.; Zheng, C.; Ji, B. Acta Chim. Sinica 2019, 77, 263. (王志强, 蔡佳林, 张明, 郑才俊, 吉保明, 化学学 报, 2019, 77, 263.)

[37] Yang, Z.; Mao, Z.; Xie, Z.; Zhang, Y.; Liu, S.; Zhao, J.; Xu, J.; Chi Z.; Aldred, M. P. Chem. Soc. Rev. 2017, 46, 915.

[38] Huang, T.; Jiang, W.; Duan, L. J. Mater. Chem. C 2018, 6, 5577.

[39] Godumala, M.; Choi, S.; Cho, M. J.; Choi, D. H. J. Mater. Chem. C 2019, 7, 2172.

[40] Cao, X.; Zhang, D.; Zhang, S.; Tao, Y.; Huang, W. J. Mater. Chem. C 2017, 5, 7699 .

[41] Godumala, M.; Choi, S.; Cho, M. J.; Choi, D. H. J. Mater. Chem. C 2016, 4, 11355 .

[42] Cai, X.; Chen, D.; Gao, K.; Gan, L.; Yin, Q.; Qiao, Z.; Chen, Z.; Jiang, X.; Su, S.-J. Adv. Funct. Mater. 2017, 27, 1704927.

[43] Bai, M.-D.; Zhang, M.; Wang, K.; Shi, Y.-Z.; Chen, J.-X.; Lin, H.; Tao, S.-L.; Zheng, C.-J.; Zhang, X.-H. Org. Electron. 2018, 62, 220. 